\title{
Oxymatrine inhibits aldosterone-induced rat cardiac fibroblast proliferation and differentiation by attenuating smad-2,-3 and-4 expression: an in vitro study
}

Lingyun $\mathrm{Fu}^{1,2+}$, Yini $\mathrm{Xu}^{2+}$, Ling Tu ${ }^{1,2}$, Haifeng Huang ${ }^{1,2}$, Yanyan Zhang ${ }^{2}$, Yan Chen ${ }^{1,2}$, Ling Tao ${ }^{2}$ and Xiangchun Shen ${ }^{1,2^{*}}$

\begin{abstract}
Background: We previously demonstrated oxymatrine, an alkaloid from the Chinese medicine radix Sophorae flavescentis, ameliorates hemodynamic disturbances and cardiac fibrosis; however, the underlying mechanisms are unclear. Here, we investigated the effect and mechanism of action of oxymatrine on aldosterone-induced cardiac fibroblast to myofibroblast differentiation in vitro.

Methods: Cardiac fibroblasts were isolated purified from neonatal Sprague Dawley rats. The optimal concentration of aldosterone to stimulate cardiac fibroblast proliferation was determined using the 3-(4,5-dimethylthiazol-2-yl)-2,5diphenyltetrazolium bromide (MTT) assay. Cardiac fibroblasts were pretreated with $7.57 \times 10^{-4} \mathrm{~mol} / \mathrm{L}$ or $3.78 \times 10^{-4}$ $\mathrm{mol} / \mathrm{L}$ oxymatrine or without oxymatrine for $2 \mathrm{~h}$, and then coincubated with $1 \times 10^{-8} \mathrm{~mol} / \mathrm{L}$ aldosterone for $48 \mathrm{~h}$. The MTT assay and Masson staining were used to detect the cardiac fibroblast proliferation and myofibroblast differentiation. The secretion of type I and III collagen was measured by commercial ELISA kits, and the hydroxyproline content was determined by the colorimetric assay. Western blotting assayed the Smad-2, Smad-3, and Smad-4 protein expression in cardiac fibroblasts.
\end{abstract}

Results: The present results confirmed that aldosterone induced cardiac fibroblast to myofibroblast proliferation and differentiation. The MTT assay and Masson staining indicated oxymatrine significantly inhibited aldosterone-induced cardiac fibroblast proliferation and myofibroblast differentiation. Oxymatrine significantly inhibited aldosterone-induced secretion of type I and III collagen, as indicated by commercial ELISA kits, and aldosterone-induced increase in hydroxyproline content, as indicated by a colorimetric assay. Western blotting revealed oxymatrine attenuated aldosterone-induced Smad-2, Smad-3, and Smad-4 expression in cardiac fibroblasts.

Conclusion: Oxymatrine can inhibit cardiac fibroblast proliferation and differentiation into myofibroblasts via a mechanism linked to attenuation of the Smad signaling pathway.

Keywords: Aldosterone, Oxymatrine, Cardiac fibroblasts, Smad-2, Smad-3, Smad-4

\footnotetext{
* Correspondence: shenxiangchun@126.com

${ }^{\dagger}$ Equal contributors

${ }^{1}$ Department of Pharmacology of Materia Medica, Guizhou Medical

University, Huaxi University town, Guian New District, Guizhou 550025, China

${ }^{2}$ The Key Laboratory of Optimal Utilization of Natural Medicinal Resources,

Guizhou Medical University, Huaxi University town, Guian new district,

Guizhou 550025, China
} 


\section{Background}

Cardiovascular diseases are a serious threat to health and are the leading cause of death in humans $[1,2]$. The mechanisms leading to cardiovascular diseases and novel drug treatments have undergone intensive research. It is well recognized that cardiac remodeling, the final pathophysiological process of cardiovascular diseases [3], is characterized by three phases: cardiomyocyte hypertrophy and apoptosis, proliferation and differentiation of cardiac fibroblasts, and extracellular matrix deposition. However, most drugs in clinical application aim to prevent cardiomyocyte hypertrophy and apoptosis, including angiotensin converting enzyme inhibitors, beta-receptor blockers and calcium antagonists. In fact, the key pathological changes during cardiac remodeling involve cardiac fibroblasts (CFs), especially CF differentiation into myofibroblasts. The differentiation of CFs results in increased secretion and deposition of myocardial collagen, which induces myocardial stiffness and myocardial diastolic and systolic dysfunction [4-6].

CFs are the main effector cells of cardiac remodeling and can proliferate and differentiate into myofibroblasts, and secrete extracellular matrix proteins such as type I and III collagen [7, 8]. The pathological process of cardiac remodeling involves a variety of factors, including the rennin-angiotensin-aldosterone system (RAAS), growth factor, transforming growth factor- $\beta$ (TGF- $\beta$ ), and nitric oxide, among others [9]. Accumulating evidence indicates that TGF- $\beta$ is the one of the key factors that promotes CF differentiation, as direct blockade of TGF- $\beta$ expression decreases extracellular matrix deposition and tissue fibrosis $[10,11]$. Increased expression of TGF- $\beta_{1}$ and Smad2/3-Smad4 are positively associated with deterioration of cardiac function after myocardial infarction $[12,13]$. The levels of CF-secreted endothelin and TGF- $\beta_{1}$ increase in cells treated with aldosterone (ALD) [14].

Oxymatrine (OMT) is one of the main bioactive ingredients of Kushen (Sophorae flavescentis radix) which is a traditional Chinese herbal medicine made from the dried root of S. flavescens Ait. (Fig. 1). Previous data demonstrated that OMT exerts a wide-range of pharmacological activities, such as anti-inflammatory, anticancer, antiviral and immune regulation effects, and it has been used to treat cardiovascular diseases [15]. Our previous study showed that OMT exerted an inhibitory effect in an experimental model of myocardial fibrosis in rats induced by acute myocardial infarction, via a mechanism involving the TGF- $\beta$-Smad signaling pathway [16]. Zhang et al. recently reported that OMT could ameliorate left ventricle hypertrophy and dysfunction in rats with heart failure [17]. Xiao and colleagues reported that OMT exerted a protective effect

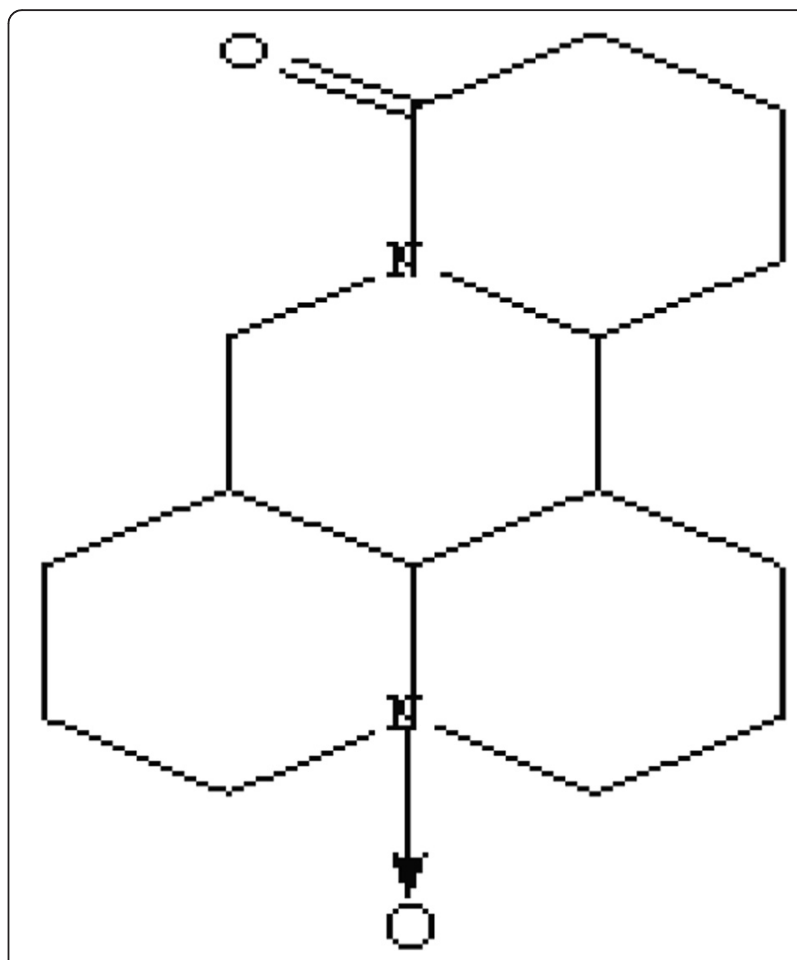

Fig. 1 Chemical structure of oxymatrine (OMT)

against ALD-mediated cardiomyocyte injury [18]. Those evidences indicate that OMT can protect the myocardium from apoptosis and fibrosis caused by a variety of stimuli. Hence, the aim of the present study was to further explore the ability of OMT to ameliorate ALD-induced apoptosis in CFs. To the best of our knowledge, this is the first demonstration that OMT protects against ALD-mediated differentiation of CFs to myofibroblasts.

\section{Methods}

\section{Ethics statement}

All animal experiments conformed to the Guide for the Care and Use of Laboratory Animals published by Guizhou Medical University and were approved by the Bioethics Committee for Animal Studies of Guizhou Medical University.

\section{Materials}

OMT (purity, 98 \%) was purchased from Green Valley Pharmaceutical Co. Ltd., Shanghai, China; ALD (purity, 98 \%) was from Fluka, Switzerland; Trypsin was from Solarbio, Beijing, China; Dulbecco's modified Eagle's medium (DMEM) was from GIBCO, Gaithersburg, USA; Penicillin and streptomycin were from Sigma, St. Louis, MO, USA; ELISA assay kits were from Dize 


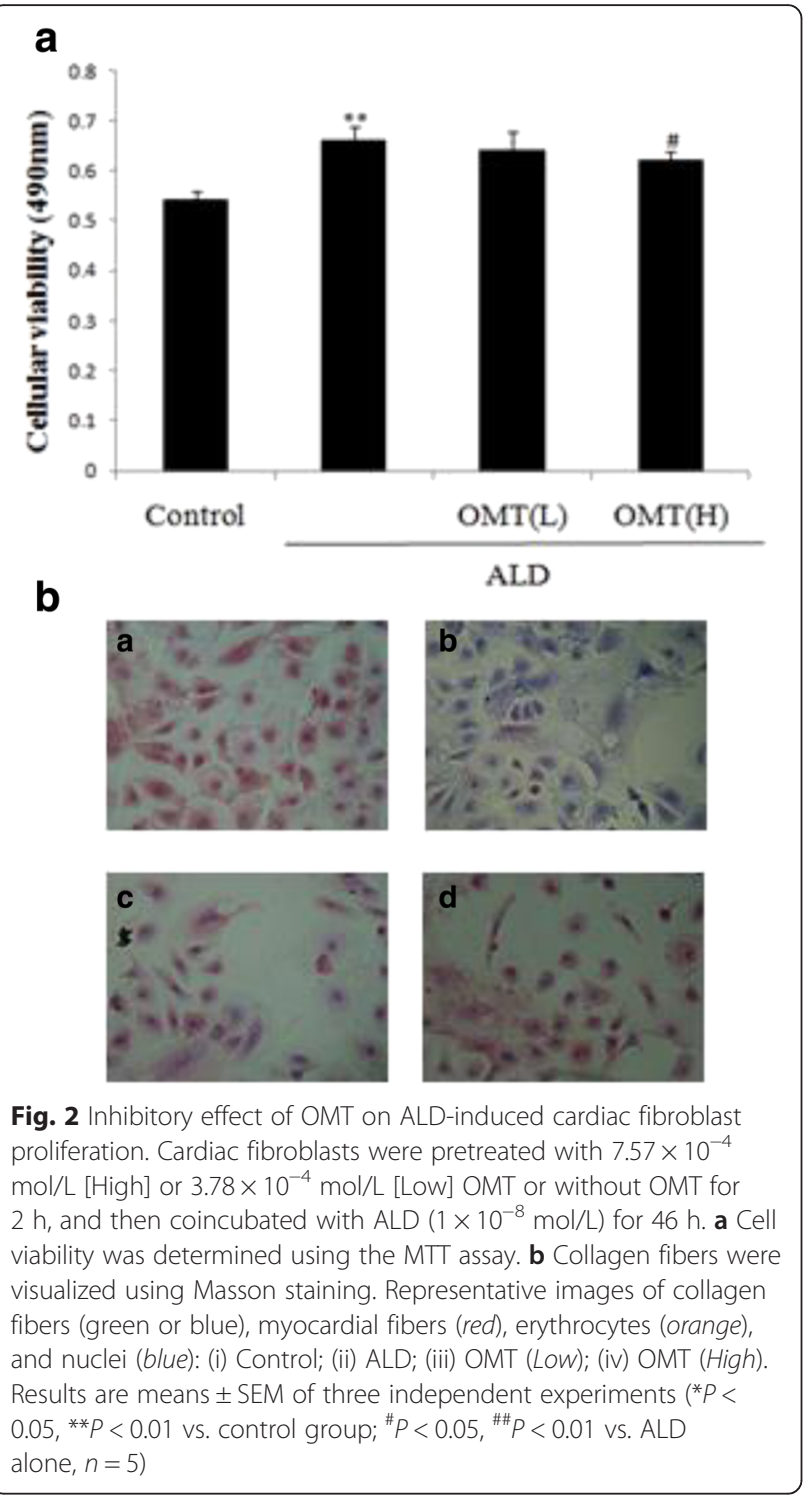

Bioengineering, Shanghai; Hydroxyproline assay kits were obtained from Jiancheng Bioengineering, Nanjing, China; and Smad-2,-3 and-4 antibodies were from Cell Signaling Technology, Beverly, USA.

\section{Isolation and culture of primary neonatal rat CFs}

CFs were isolated and purified from 1- to 3-day-old Sprague-Dawley rats. Briefly, the hearts of 1-3 day-old Sprague Dawley rats were isolated and digested in $10 \mathrm{~mL}$ of phosphate buffered saline (PBS; $137 \mathrm{mM} \mathrm{NaCl}$,

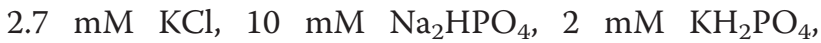
$\mathrm{pH} 7.2-7.4)$ containing $0.08 \%$ trypsin for $10 \mathrm{~min}$ at $37{ }^{\circ} \mathrm{C}$. After each digestion step, the medium containing suspended cells was removed and an equal volume of Spinner/collagenase solution was added.
Primary cultures of rat cardiac stromal cells were grown in DMEM supplemented with $20 \%$ fetal bovine serum, penicillin $(100 \mathrm{U} / \mathrm{mL})$ and streptomycin $(100 \mathrm{U} / \mathrm{mL})$ at $37{ }^{\circ} \mathrm{C}$ in a humidified atmosphere of $5 \% \mathrm{CO}_{2}$. CFs at the third or fourth passage was used for experiments. The seeding density was $1 \times 10^{5}$ cells/mL for the MTT assay and morphological analyses and $2 \times 10^{5}$ cells $/ \mathrm{mL}$ for Western blot analysis. The purity of the neonatal rat CF cultures was about $99 \%$, as indicated by vimentin immunocytochemical staining.

\section{CF proliferation assay}

CFs cultured in 96-well plates were exposed to ALD $\left(1 \times 10^{-8} \mathrm{M}\right)$ alone for $48 \mathrm{~h}$ or pretreated with different concentrations of OMT $\left(3.78 \times 10^{-4} \mathrm{M}\right.$ to $\left.7.57 \times 10^{-4} \mathrm{M}\right)$ for $2 \mathrm{~h}$ before exposure to ALD for $48 \mathrm{~h}$. Then, 3-(4,5dimethyl-thiazol-2-yl)-2,5-diphenyltetrazolium bromide (MTT) was added to each well (final concentration $0.5 \mathrm{mg} / \mathrm{mL}$ ) in sterile conditions, and the plates were incubated for $4 \mathrm{~h}$ at $37{ }^{\circ} \mathrm{C}$ in a $5 \% \mathrm{CO}_{2}$ incubator, finally the medium was discarded and washed 3 times with PBS. Formazan salt crystals were dissolved by addition of $150 \mu \mathrm{L}$ dimethylsulfoxide per well and the absorbance values were determined at $490 \mathrm{~nm}$ using a microplate reader (ELX800; GE, USA).

\section{Enzyme-linked immunosorbent assay (ELISA)}

The levels of type I and III collagen in the cell lysis buffer and cell supernatants were measured using ELISA assay kits. The OD values were measured at $450 \mathrm{~nm}$ using an ELX800 microplate reader.

\section{Hydroxyproline colorimetric assay}

The hydroxyproline (Hyp) content of the cell lysis buffer and cell supernatants was quantified using a commercial Hyp detection kit. The OD values of the samples were measured at $550 \mathrm{~nm}$ using an ELX800 microplate reader.

\section{Western blotting}

Western blotting assays were used to measure the protein expression levels of Smad-2,-3,-4, and $\beta$-actin in CFs. After treatment, CFs were washed once in ice-cold PBS, and then lysed in lysis buffer (Dingguo, Beijing, China) on ice. Protein concentrations were assessed using a bicinchoninic acid protein assay kit (Dingguo, Beijing, China). Equal amounts of protein were subjected to $12 \%$ SDS-polyacrylamide gel electrophoresis, transferred onto PVDF membranes using a Bio-Rad Western blot analysis apparatus, and then the membranes were blocked in $5 \%$ non-fat dry milk in TBST, then incubated with primary Smad-2,-3,-4 (1:1000 dilution), and $\beta$-actin (1:1000; Cell Signaling 
Technology) antibodies overnight at $4{ }^{\circ} \mathrm{C}$. After washing three times with TBST, the membranes were incubated with the corresponding secondary antibodies (1:4000, Sigma, MS, USA) for $2 \mathrm{~h}$ at room temperature, and the immunolabeled bands were visualized using Pierce ECL Western blotting substrate (Millipore, Bedford, USA).

\section{Statistical analysis}

All data are presented as the mean \pm SEM. Betweengroup comparisons were performed using $t$-tests. All data analysis was performed using Microsoft Excel. Statistical significance was defined as $P<0.05 ; P<0.01$ was considered highly significant.

\section{Results}

\section{OMT inhibits ALD-induced CF proliferation and}

\section{differentiation}

The MTT assay and Masson staining were used to assess the ability of OMT to inhibit ALD-induced CF proliferation and differentiation. CFs were pretreated with or without different concentrations of OMT $\left(3.78 \times 10^{-4} \mathrm{M}\right.$ and $\left.7.57 \times 10^{-4} \mathrm{M}\right)$ for $2 \mathrm{~h}$ and then stimulated with ALD $\left(1 \times 10^{-8} \mathrm{M}\right)$ for $24 \mathrm{~h}$. The MTT assay showed that ALD significantly increased CF proliferation compared to control cells.
However, pretreatment with OMT inhibited the ALD-induced increase in CF proliferation (Fig. 2a). CF differentiation was also investigated using Masson staining (Fig. 2b). ALD increased CFs differentiation to myofibroblasts and collagen deposition compared to control cells; however, OMT significantly ameliorated ALD-induced CF differentiation and collagen deposition.

\section{OMT attenuates ALD-induced type collagen I and colla-} gen III deposition in CFs

Type I and III collagen are the key biomarkers of CFs differentiation into myofibroblasts. ELISAs were used to assess the secretion of type I and III collagen. Exposure of CFs to ALD significantly increased the levels of type I and III collagen in the cell lysis buffer and cell supernatant compared to control cells (Fig. 3a-d). However, pretreatment with OMT attenuated the ALD-induced increases in the levels of type I and III collagen in the cell lysis buffer and cell supernatant (Fig. 3a-d).

\section{OMT inhibits ALD-induced hydroxyproline secretion by CFs}

Hyp, a degradation production of collagen, represents an index of collagen secretion which, to an
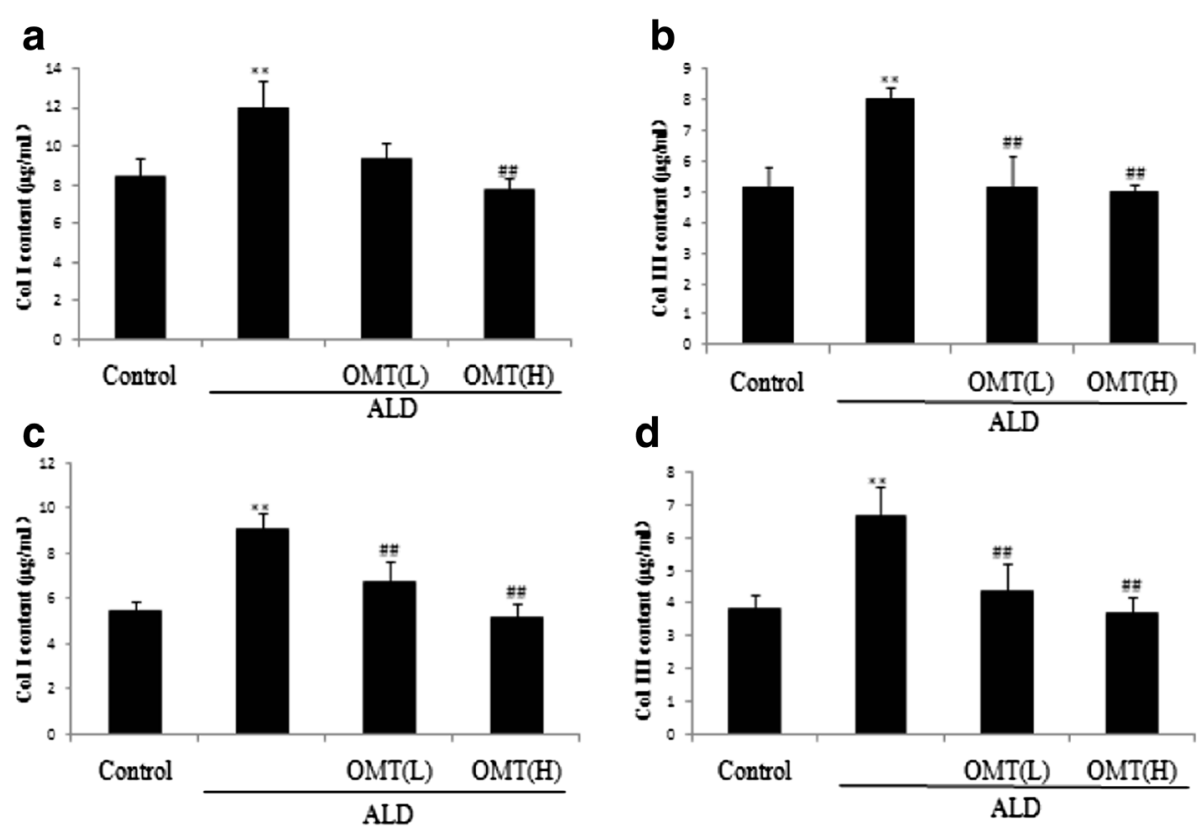

Fig. 3 Inhibitory effects of OMT on ALD-induced type I and III collagen secretion of cardiac fibroblasts. Cardiac fibroblasts were pre-treated with $7.57 \times 10^{-4} \mathrm{~mol} / \mathrm{L}[\mathrm{High}]$ or $3.78 \times 10^{-4} \mathrm{~mol} / \mathrm{L}$ [Low] OMT or without OMT for $2 \mathrm{~h}$, and then coincubated with ALD $\left(1 \times 10^{-8} \mathrm{~mol} / \mathrm{L}\right)$ for $46 \mathrm{~h}$. a ELISA assays of type I collagen content in cell lysis buffer; (b) type III collagen content in cell lysis buffer; (c) type I collagen content in cell supernatant; and (d) type III collagen content in cell supernatant. Results are means \pm SEM of three independent experiments. ${ }^{*} P<0.05$, ${ }^{* *} P<0.01$ vs. control cells; ${ }^{\#} P<0.05,{ }^{\# \#} P<0.01$ vs. ALD alone, $n=5$ ) 
extent, can reflect myofibroblast secretion activity. Hyp was measured using a commercial colorimetric assay. ALD increased the Hyp content of the cell lysis buffer and cell supernatant of cultured CFs (Fig. 4a-b). Compared to cells treated with ALD, pretreatment with OMT significantly reduced the ALDinduced increase in the Hyp content of the cell lysis buffer and cell supernatant (Fig. 4a-b).

\section{OMT inhibits ALD-induced expression of smad-2,-3, and-4} in CFs

The TGF- $\beta$-Smad signaling pathway provides the strongest activation signal for organic fibrosis; Smad-2,-3, and-4 are key factors in this signaling pathway. Therefore, we explored Smads protein expression in CFs exposed to ALD. Western blotting showed that ALD enhanced the expression of Smad-2,-3, and-4 in CFs (Fig. 5a-c) compared to control cells. Pretreatment with

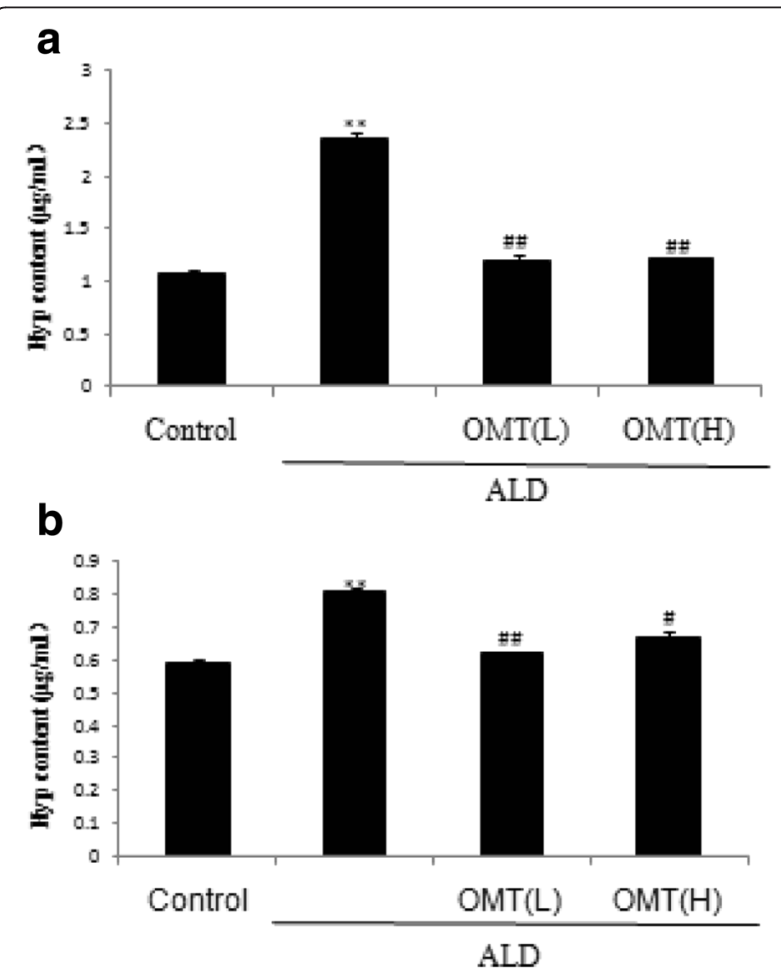

Fig. 4 Inhibitory effect of OMT on ALD-induced hydroxyproline (Hyp) secretion by cardiac fibroblasts. Cardiac fibroblasts were pre-treated with $7.57 \times 10^{-4} \mathrm{~mol} / \mathrm{L}$ [High] or $3.78 \times 10^{-4} \mathrm{~mol} / \mathrm{L}$ [Low] OMT or without OMT for $2 \mathrm{~h}$, and then coincubated with ALD $\left(1 \times 10^{-8} \mathrm{~mol} / \mathrm{L}\right)$ for $46 \mathrm{~h}$. a Analysis of hydroxyproline content in cell lysis buffer and (b) cell supernatant. Results are means \pm SEM of three independent experiments. $\left({ }^{*} P<0.05,{ }^{* *} P<0.01\right.$ vs. control cells; ${ }^{\#} P<0.05,{ }^{\# \#} P<0.01$ vs. ALD alone, $n=4$ )
OMT attenuated the ALD-induced increases in Smad-2,3 , and-4 protein expressions in CFs.

\section{Discussion}

Chronic heart failure is a pathological process caused by cardiac remodeling events, including myocardial hypertrophy, myocardial cell loss, and myocardial fibrosis (MF) [19-21]. Clinical data have confirmed MF occurs as an inevitable process during the progression of heart disease to its terminal stage and the key factors of cardiac function by compensatory period to decompensation period [22, 23]. Therefore, inhibition of abnormal cardiac remodeling, the reaction to target organ damage and fibrosis, by reversing a variety of chronic inflammatory reactions, may represent a primary treatment strategy to improve clinical outcomes and reduce mortality in patients with chronic heart failure [24].

It has been confirmed that the RAAS is a key signal transduction pathway involved in organic fibrosis. Recent reports have indicated that ALD, the final molecule of the RAAS pathway, is widely implicated in myocardial fibrosis and promotes the synthesis of collagen in cardiac CFs [25-28]. In this study, to contribute to the development of novel therapeutic strategies for treating cardiovascular disease, we investigated the ability of OMT to inhibit ALD-induced CF proliferation and differentiation in vitro and explored the associated mechanisms.

The MTT assay showed that CFs exposed to $1 \times 10^{-8}$ $\mathrm{mol} / \mathrm{L}$ ALD alone for $48 \mathrm{~h}$ displayed significantly higher levels of proliferation $(P<0.01)$ compared with control cells. However, pretreatment with OMT significantly attenuated ALD-induced cell proliferation. Masson staining confirmed that OMT significantly reduced ALDinduced collagen fiber accumulation in CFs, and ELISAs suggested that OMT inhibited the ALD-induced secretion of type I collagen, type III collagen and Hyp.

Smad-2,-3, and-4, and the TGF- $\beta 1$-Smads pathway are implicated in fibrosis. Activation of TGF- $\beta 1$ Smads is an important signal that leads to cardiac fibrosis. Western blotting showed ALD significantly increased the expression of Smad-2,-3, and-4; these three proteins can promote myocardial fibrosis and play major roles in the TGF- $\beta$-Smad signaling pathway. However, OMT significantly inhibited ALDinduced protein expression of Smad-2,-3 and-4 compared to cells treated with ALD alone. These results suggest that OMT may inhibit ALD-induced proliferation and differentiation of CFs via a mechanism linked to the TGF- $\beta /$ Smad signaling pathway and downregulation of Smad-2,-3 and-4 protein expression. The potential of OMT as a drug to prevent and treat MF merits further research in preclinical models. 

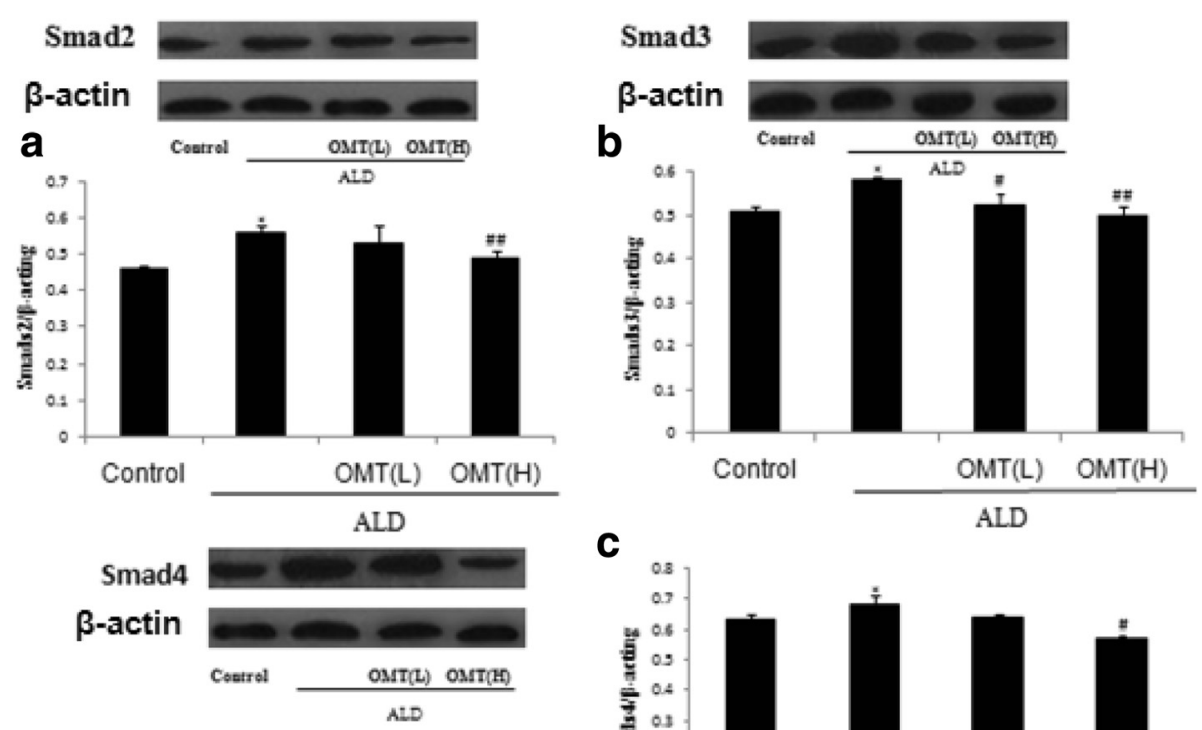

C

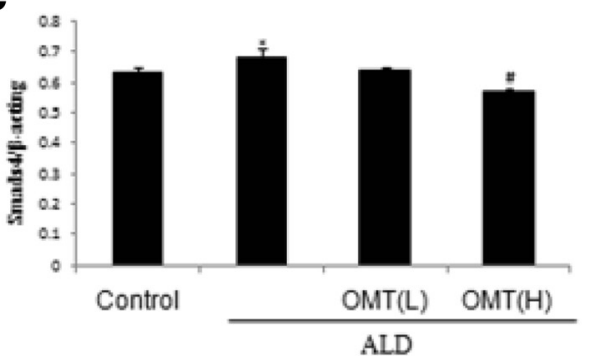

Fig. 5 Inhibitory effect of OMT on ALD-induced expression of Smad-2,-3, and-4 in cardiac fibroblasts. Cardiac fibroblasts were pretreated with $7.57 \times 10^{-4} \mathrm{~mol} / \mathrm{L}$ [High] or $3.78 \times 10^{-4} \mathrm{~mol} / \mathrm{L}$ [Low] OMT or without OMT for $2 \mathrm{~h}$, and then coincubated with ALD $\left(1 \times 10^{-8} \mathrm{~mol} / \mathrm{L}\right) \mathrm{for} 46 \mathrm{~h}$. Western blotting analysis of (a) Smad-2, (b) Smad-3 and (c) Smad-4. Results are means \pm SEM of three independent experiments. $\left({ }^{*} P<0.05,{ }^{* *} P<0.01\right.$ vs. control cells; ${ }^{\#} P<0.05,{ }^{\# \#} P<0.01$ vs. ALD alone, $\left.n=4\right)$

\section{Conclusion}

OMT attenuates ALD-induced CF proliferation and differentiation into myofibroblasts via a mechanism that involves the TGF- $\beta$-Smad signal transduction pathway. The present study highlights on a novel molecular mechanism by which OMT inhibits ALD-induced CF differentiation into myofibroblasts.

\section{Abbreviations}

ALD, aldosterone; CFs, cardiac fibroblasts; DMEM, dulbecco's modified Eagle's medium; Hyp, hydroxyproline; MF, myocardial fibrosis; MTT, 3-(4,5dimethylthiazol-2-yl)-2,5-diphenyltetrazolium bromide; ; OMT, oxymatrine; PBS, phosphate buffered saline; RAAS, rennin-angiotensin-aldosterone system; TGF- $\beta$, transforming growth factor- $\beta$

\section{Acknowledgments}

The authors would like to acknowledge the National Natural Science Foundation of China, the Key Project for Science and Technology Foundation of Guizhou Province, the Innovated Team of the Education Department of Guizhou Province, the Scientific and Technologic Innovated Team of Guizhou Province, the Program for New Century Excellent Talents in University, the High Level Innovation Talents, the 2011 Modern Drug of Cooperation Innovation, and the scientific and technologic cooperation project of Guizhou province and Guiyang medical University. In addition, we thank Guizhou Medical University for providing the necessary facilities.

\section{Funding}

This research was supported by grants from the National Natural Science Foundation of China (No. 81173586, 81560588), the Key Project for Science and Technology Foundation of Guizhou Province (No. JZ2015-2039), the Innovated Team of the Education Department of Guizhou Province (No. 2014-31), the Scientific and Technologic
Innovated Team of Guizhou Province (No. 2015-4025), the Program for New Century Excellent Talents in University (NCET-13-0747), the High Level Innovation Talents (No.2015-4029), the 2011 Modern Drug of Cooperation Innovation (No.[2013]04), and the scientific and technologic cooperation project of Guizhou province and Guiyang medical University (No. LH-2014-7098).

\section{Availability of data and materials}

The datasets supporting the conclusions of this article are included within the article.

\section{Authors' contributions}

SXC were responsible for the design of study and providing research fund. SXC and TL analyzed the data and provided technical guidance. $F L Y$ and $X Y N$ made experimental operation and wrote the papers. $T L$, HHF, ZYY, CY and TL participated in cell experimental and statistical analysis. All authors read and approved the final manuscript.

\section{Competing interests}

The authors declare that they have no competing interests.

\section{Consent for publication}

This information is not relevant.

\section{Ethics approval and consent to participate}

All animal experiments conformed to the Guide for the Care and Use of Laboratory Animals published by Guizhou Medical University and was approved by the Bioethics Committee for Animal Studies of Guizhou Medical University.

Received: 23 December 2015 Accepted: 19 July 2016

Published online: 26 July 2016 


\section{References}

1. Wang CC, Tu YF, Yu ZL, Lu RZ. PM2.5 and Cardiovascular Diseases in the Elderly: An Overview. Int. J. Environ. Res. Public Health. 2015;12:8187-97.

2. Ebert $A D$, Diecke $S$, Chen IY, Wu JC. Reprogramming and transdifferentiation for cardiovascular development and regenerative medicine: where do we stand. EMBO Mol Med. 2015;7:1-14.

3. Cannon MV, Silljé HHW, Sijbesma JWA, Baudoin IV, Ciapaite J, Sluis BVD, et al. Cardiac LXRa protects against pathological cardiac hypertrophy and dysfunction by enhancing glucose uptake and utilization. EMBO Mol Med. 2015:5:1-15.

4. Doltra A, Messroghli D, Stawowy P, Hassel JH, Gebker R, Leppanen O, et al. Potential Reduction of Interstitial Myocardial Fibrosis With Renal Denervation. J Am Heart Assoc. 2014;3:1-10.

5. Meng GL, Zhu JB, Xiao YJ, Huang ZR, Zhang YQ, Tang X, et al. Hydrogen Sulfide Donor GYY4137 Protects against Myocardial Fibrosis. Oxidative Medicine Cellular Longevity. 2015;15:1-10

6. Dadson K, Turdi S, Boo S, Hinz B, Sweeney G. Temporal and Molecular Analyses of Cardiac Extracellular Matrix Remodeling following Pressure Overload in Adiponectin Deficient Mice. Plos one. 2015;10:1-20.

7. Rosin NL, Gareau AJ, Betsch D, Falkenham A, Sopel MJ, Lee TD, et al. Antibody therapy can enhance Angiotensinll-induced myocardial fibrosis. Fibrogenesis \& Tissue Repair. 2014;7:1-9.

8. Pichler M, Rainer PP, Schauer S, Hoefler G. Cardiac Fibrosis in Human Transplanted Hearts Is Mainly Driven by Cells of Intracardiac Origin. J Am Coll Cardiol. 2012;11:1008-16.

9. Li ZZ, Jiang H, Chen D, Liu Q, Geng J, Guo JQ, et al. Renal Sympathetic Denervation Improves Cardiac Dysfunction in Rats with Chronic Pressure Overload. Physiological Research Pre-Press Article. 2015;4:1-23.

10. Ghavami S, Cunnington RH, Gupta S, Yeganeh B, Filomeno KL, Freed DH, et al. Autophagy is a regulator of TGF- $\beta 1$-induced fibrogenesis in primary human atrial myofibroblasts. Cell Death and Disease. 2015;6:1-11.

11. Sun LL, Jin HF, Sun LJ, Chen SY, Huang YQ, Liu J, et al. Hydrogen Sulfide Alleviates Myocardial Collagen Remodeling in Association with Inhibition of TGF- $3 /$ Smad Signaling Pathway in Spontaneously Hypertensive Rats. Mol Med. 2014;20:503-15.

12. Chen Y, Yang SJ, Yao WJ, Zhu HY, Meng GL, Zhang W. Prostacyclin Analogue Beraprost Inhibits Cardiac Fibroblast Proliferation Depending on Prostacyclin Receptor Activation through a TGFß-Smad Signal Pathway. Plos one. 2014;9:1-14.

13. Schwartze JT, Becker S, Sakkas E, Wujak LA, Niess G, Usemann J, et al. Glucocorticoids Recruit Tgfbr3 and Smad1 to Shift Transforming Growth Factor- $\beta$ Signaling from the Tgfbr1/Smad2/3 Axis to the Acvrl1/Smad1 Axis in Lung Fibroblasts. J Biol Chem. 2014;5:3262-75.

14. Bunda S, Wang YT, Mitts TF, Liu P, Arab S, Arabkhari M, et al. Aldosterone Stimulates Elastogenesis in Cardiac Fibroblasts via Mineralocorticoid Receptor-independent Action Involving the Consecutive Activation of Ga13, c-Src, the Insulin-like Growth Factor-I Receptor, and Phosphatidylinositol 3Kinase/Akt. The Journal Of Biological Chemistry. 2015;24:16633-47.

15. Guzman JR, Koo JS, Goldsmith JR, Muhlbauer M, Narular A, Jobin C. Oxymatrine Prevents NF-kB Nuclear Translocation And Ameliorates Acute Intestinal Inflammation. Sci Rep. 2013:3:1-9.

16. Shen XC, Yang YP, Xiao TT, Peng J, Liu XD. Protectiv effect of oxymatrine on myocardial fibrosis induced by acute myocardial infarction in rats involved in TGF- $\beta 1-S m a d s$ signal pathway. J Asian Nat Prod Res. 2011;3:215-24.

17. Zhang W, Zhang J, Kang YS, Liu JJ, Wang XQ, Xu QB, et al. Cardioprotective effects of oxymatrine on isoproterenol-induced heart failure via regulation of DDAH/ADMA metabolism pathway in rats. Eur J Pharmacol. 2014;745:29-35.

18. Xiao T, Wang YY, Zhang Y, Bai CH, Shen XC. Similar to Spironolactone, Oxymatrine Is Protective in Aldosterone-Induced Cardiomyocyte Injury via Inhibition of Calpain and Apoptosis-Inducing Factor Signaling. PLoS One. 2014;9:e88856-61.

19. Zhu XQ, Hong HS, Lin XH, Chen LL, Li YH. Changes in cardiac aldosterone and its synthase in rats with chronic heart failure: an intervention study of long-term treatment with recombinant human brain natriuretic peptide. Braz J Med Biol Res. 2014;47:646-54

20. Červenka L, Melenovský V, Husková Z, Sporková A, Bürgelová M, Škaroupková $P$, et al. Inhibition of soluble epoxide hydrolase does not improve the course of congestive heart failure and the development of renal dysfunction in rats with volume overload induced by aorto-caval fistula. Physiological Research Pre-press Article. 2015:4:1-38.
21. Vianello A, Caponi L, Galetta F, Franzoni F, Taddei M, Rossi M, et al. $\beta 2$ -Microglobulin and TIMP1 Are Linked Together in Cardiorenal Remodeling and Failure. Cardiorenal Medicine. 2015;5:1-11.

22. Chu PY, Walder K, Horlock D, Williams D, Nelson E, Byrne M, et al. CXCR4 Antagonism Attenuates the Development of Diabetic Cardiac Fibrosis. Plos one. 2015:7:1-13.

23. SamuliLepojärvi E, Piira OP, Pääkkö E, Lammentausta E, Risteli J, Miettinen JA, et al. SerumPINP, PIIINP, galectin-3, and ST2 as surrogates of myocardial fibrosis and echocardiographic left ventricular diastolic filling properties. Original Research. 2015;6:1-6.

24. Jacobi J, Maas R, Cordasic N, Koch K, Schmieder RE, Boger RH, et al. Role of asymmetric dimethylarginine for angiotensin Il-induced target organ damage in mice. Am J Physiol Heart Circ Physiol. 2007;5:H1058-66.

25. Boulkroun S, Fernandes-Rosa FL, Zennaro MC. Molecular and cellular mechanisms of aldosterone producing adenoma development. Front Endocrinol. 2015;6:1-8.

26. Singh JS, Lang CC. Angiotensin receptor-neprilysin inhibitors: clinical potential in heart failure and beyond. Vasc Health Risk Manag. 2015;4:283-95.

27. Zhang SH, Wang J, Jin TR, Zhang LX, Shao J. The role of spironolactone in the metabolism of serum type I collagen in elderly patients with atrial fibrillation. Eur Rev Med Pharmacol Sci. 2014;18:2903-7.

28. Maron BA, Oldham WM, Vargas SO, Arons E, Zhang YY, Loscalzo J, et al. Upregulation of Steroidogenic Acute Regulatory Protein by Hypoxia Stimulates Aldosterone Synthesis in Pulmonary Artery Endothelial Cells to Promote Pulmonary Vascular Fibrosis. Circulation. 2014;2:168-79.

\section{Submit your next manuscript to BioMed Central and we will help you at every step:}

- We accept pre-submission inquiries

- Our selector tool helps you to find the most relevant journal

- We provide round the clock customer support

- Convenient online submission

- Thorough peer review

- Inclusion in PubMed and all major indexing services

- Maximum visibility for your research

Submit your manuscript at www.biomedcentral.com/submit 